Maciej Serowaniec

Nicolaus Copernicus University: Toruń, Poland

mserowaniec@umk.pl

ORCID iD: https://orcid.org/0000-0003-4693-7977

ORCID iD: https://orcid.org/0000-0002-7595-0575

ZBIGNIEW WITKOWSKI

Nicolaus Copernicus University: Toruń, Poland

zbywit@umk.pl

ORCID iD: https://orcid.org/0000-0003-3220-2697

\title{
THE EROSION OF THE LEGISLATIVE PROCESS In Poland
}

\section{L'EROSIONE DELLA FUNZIONE LEGISLATIVA IN Polonia}

\begin{abstract}
In conformità a quanto disposto dallart. 10, comma 2 della Costituzione della Repubblica di Polonia la funzione legislativa è attribuita al parlamento. Da alcuni anni, il procedimento di formazione delle leggi conosce una serie di sistematiche irregolarità legate a: cambiamenti troppo frequenti della situazione giuridica, strumentalizzazione della consultazione pubblica e una eccessiva rapidità del processo di redazione e di approvazione delle leggi, comportando una bassa qualità delle stesse. Nell'ultimo legislatura, la situazione non è migliorata: per molti aspetti i problemi esistenti si sono intensificati e gli standard del processo legislativo si sono drammaticamente abbassati. Il Parlamento è diventato una 'manifattura' delle leggi in cui tutto può essere 'prodotto'.

\section{SUMMARY}

In accordance with the provisions of Article 10 paragraph 2 of the Constitution of the Republic of Poland, the basic function of the Polish Parliament is the legislative function. However, for years in the field of law making there have been a number of irregularities related with, among others too frequent changes in the legal status,
\end{abstract}


the adverse treatment of public consultations, and with undue haste in law making, which all translate into its low quality. During the last parliamentary term the state of affairs in this field has not improved and in many respects the existing problems have intensified. In consequence, the standards of the Polish law making process have deteriorated. The Parliament has become a "manufacture" of law in which everything can be "produced".

Parola Chiave: funzione legislativa, parlamento, processo legislativo, Costituzione della Repubblica di Polonia.

KeYwORDs: legislative process, parliament, law making process, Constitution of the Republic of Poland.

\section{INTRODUZIONE}

Nell'ultimo legislatura della Camera dei Deputati e del Senato della Repubblica di Polonia, possiamo osservare un intensificarsi di fenomeni negativi: si ricorre sempre più spesso a procedimenti legislativi abbreviati, il tempo previsto per le consultazioni viene abbreviato, non si prendono in considerazione i pareri e le obiezioni sollevate, i progetti governativi di legge vengono presentati come proposte dei deputati, si elaborano relazioni schematiche su consultazioni svolte che non aggiungono alcunché alla materia analizzata e si assiste a un abbondono quasi assoluto di audizioni pubbliche (A. Antoszewski, 2018, p. 13).

Le leggi hanno smesso di rappresentare le regole della ragione (logos) che consentono di modellare l'ordine sociale. La legge è diventata ciò che la maggioranza parlamentare considera legge, mentre dovrebbe seguire le modalità definite dalla costituzione per la sua formazione, conformarsi materialmente alla costituzione e allo stesso tempo soddisfare il requisito della razionalità (M. Zubik, 2020, pp. 731-732). Dovrebbe servire all'efficace attuazione degli obiettivi prefissati. Questo requisito è impossibile da realizzare se la legge è scritta senza avere riguardo alle regole di drafting di base e senza rispetto delle procedure costituzionalmente prescritte; se durante il processo legislativo e alla conclusione dello stesso l'impatto di una data norma viene valutato superficialmente e senza un'istruttoria appropriata (M. Wyrzykowski, 2020, pp. 721-722). 


\section{Procedimenti legislativi ABbreviati}

Naturalmente, in casi specifici, a causa della natura urgente di alcuni problemi, l'ordinamento polacco prevede l'uso di procedimenti legislativi di natura abbreviata. Ma in pratica, il Consiglio dei Ministri e i deputati vi ricorrono liberamente, tentando soprattutto di limitare la necessità di concordare, consultare ed esprimere pareri su progetti di legge. Conformemente a quanto disposto dal regolamento del Consiglio dei Ministri (Monitor Polski 2013 voce 979, con successive modifiche). tutti i progetti di atti governativi dovrebbero essere accordati, consultati tramite consultazioni pubbliche e adeguatamente valutati. Qualora l'importanza o l'urgenza della questione richiedano la sua immediata risoluzione da parte del Consiglio dei Ministri, il Primo Ministro può decidere di applicare il cosiddetto procedimento abbreviato (artt.98-99 del regolamento). Gli atti governativi elaborati secondo questa procedura non richiedono l'applicazione di tutti gli elementi del processo legislativo nei tempi previsti, come concertazioni, consultazioni pubbliche e valutazioni, nonché l'esame del progetto da parte del Comitato Permanente del Consiglio dei Ministri o da una commissione giuridica.

Nell'attuale legislatura, si è ricorsi al procedimento abbreviato per un quarto dei disegni di legge governativi resi pubblici sulla piattaforma del Centro Governativo di Legislazione. Dall'analisi dei disegni di legge presentati si evince tuttavia che la maggior parte di essi non rappresentava una reazione a situazioni straordinarie. A giudicare dalle discussioni che tali disegni di legge hanno suscitato durante i lavori in parlamento, labbandono di consultazioni e valutazioni non era giustificato. Altrettanto importante rimane il fatto che nei documenti pubblicati sulla piattaforma del Centro Governativo di Legislazione in merito a questi progetti, nella stragrande maggioranza dei casi, non è stata allegata alcuna lettera comprovante il consenso del Primo Ministro per l'adozione del procedimento abbreviato.

Il procedimento abbreviato è previsto dall'art. 51 del regolamento della Camera dei Deputati (Delibera della Camera dei Deputati della Repubblica di Polonia del 30 luglio 1992, Monitor Polski 1992, n.26, voce 185, con successive modifiche). Conformemente a quanto disposto, in casi giustificati, la Camera dei Deputati può abbreviare i procedimenti e procedere alle fasi successive appena i deputati ricevono il dossier inerente a un dato progetto di legge. 
È interessante notare che tra i progetti di legge presentati nell'attuale legislatura non ce ne sia stato nessuno formalmente esaminato con il procedimento abbreviato. Tuttavia, in pratica, si è ricorso di fatto al medesimo in diversi casi. Il procedimento abbreviato (i lavori sono durati meno di 14 giorni) è stato adottato dalla Camera dei Deputati ad esempio nel caso del progetto di legge sul Centro di Trasporto Nazionale (3 giorni, Documento parlamentare n. 2473), del progetto di legge volto a modificare la legge del 27 luglio 2001 sullorganizzazione dei tribunali ordinari e la legge dell'8 dicembre 2017 sulla Corte suprema (1 giorno, Documento parlamentare n. 2389) e della proposta di legge, di iniziativa dei deputati, volta a modificare la Legge sulla Corte suprema e alcune altre leggi (2 giorni, Documento parlamentare n. 2480). Tuttavia, conformemente al regolamento della Camera dei Deputati, il tempo minimo dei lavori su un progetto non può essere inferiore a 14 giorni. La prima lettura del progetto può avere luogo non prima del settimo giorno, successivo alla consegna del progetto ai deputati (art. 37, comma 4), mentre la seconda lettura può avere luogo non prima del settimo giorno dalla data di consegna del rapporto elaborato dalla commissione (art. 44, comma 3). La terza lettura invece può avere luogo immediatamente dopo la seconda lettura del progetto, qualora nel frattempo il progetto non sia stato rinviato in commissione (art. 48).

Indipendentemente dalla tipologia di procedimento adottato ogni volta dalla Camera dei Deputati, la terza lettura effettuata quasi immediatamente dopo la seconda lettura è diventata praticamente una prassi. Nel caso, ad esempio, del disegno di legge di iniziativa del governo inerente la modifica della legge sull'Istituto Polacco per la Memoria Nazionale - Commissione per la lotta ai reati commessi contro la nazione polacca, del disegno di legge sulla gestione collettiva di diritti d'autore e diritti connessi (in questo caso la seconda lettura, la riunione della commissione e la terza lettura si sono svolte in un giorno solo) o del disegno di legge sempre di iniziativa del governo sulla modifica della legge in materia di sistema di previdenza sociale e di alcune altre leggi, l'intervallo tra le due letture è durato solo qualche ora.

Un vero simbolo della strumentalizzazione del ruolo del parlamento, di fatto subordinato al centro decisionale esterno, sono diventati i lavori dedicati alla modifica della legge sull'Istituto Polacco per la Memoria 
Nazionale. Lapprovazione della legge di modifica, unitamente alla firma del capo di stato, in quel periodo in visita ufficiale all'estero, si è conclusa in poche ore. Il progetto di legge è stato presentato alla Camera dei Deputati il 27 giugno 2018 alle ore 8. Dopo unora e mezza, è iniziata la prima lettura. A ciascun deputato, intenzionato a presentare commenti o riserve, è stato consentito 1 minuto di tempo. In seguito, senza alcuna discussione si sono svolte la seconda e la terza lettura. Dopo due ore, alle 11.29, il progetto è stato deliberato e approvato. I lavori del Senato a sua volta sono durati meno di cinque ore. In segno di protesta per la limitazione fino a soli cinque minuti, del tempo consentito ai senatori durante la plenaria, i senatori dellopposizione hanno lasciato l'aula. Il processo legislativo si è concluso intorno alle ore 18:00, quando il Presidente della Repubblica, in visita ufficiale in Lettonia, ha apposto sulla legge una firma elettronica. Dalla data di presentazione alla Camera dei Deputati della proposta di legge d'iniziativa dei deputati, volta a modificare la legge sulla Procura della Repubblica e alcune altre leggi, sono trascorsi invece nove giorni, ma la Camera dei Deputati ha lavorato su detto progetto un giorno e mezzo. Si è trattato di un disegno di legge che ha modificato di diverse leggi, alla fine intitolato "legge che modifica la Legge sullorganizzazione dei tribunali ordinari e alcune altre leggi” (G. Markowski, 2020).

La maggioranza dei deputati ha ignorato lopinione molto critica del Difensore Civico, il quale può esprimersi, d'ufficio, su ogni progetto di legge? che ha scritto: "Lanalisi dell'intero disegno di legge porta alla conclusione che il medesimo è stato preparato non come risposta ad attuali problemi della giustizia bensì rappresenta una risposta alla situazione attuale, sorta a seguito dell'adozione delle leggi sulla magistratura, che nell'opinione di molte istituzioni e organismi internazionali (Commissione di Venezia, Commissione Europea, Ufficio per le istituzioni democratiche e i diritti umani "ODIHR", il relatore speciale delle Nazioni Unite, Gruppo di Stati contro la corruzione "GRECO") non erano conformi agli standard internazionali. L'implementazione di cambiamenti significativi alle leggi, attraverso le proposte di legge da parte dei deputati, che hanno consentito di aggirare gli accordi e le consultazioni pubbliche, deve essere giudicata negativamente. La modalità di lavorare sul progetto di legge è risultata estremamente veloce" (Documento parlamentare n. 2731). 
Nel dibattito pubblico sono sorti anche molti dubbi circa la correttezza della modalità di lavoro adottata dalla Camera dei Deputati, durante il dibattito avvenuto il 16 dicembre 2016 sulla legge di bilancio. A nostro avviso, esistono seri dubbi sulla sua conformità alla Costituzione e ai regolamenti della Camera dei Deputati. Questi dubbi riguardano, tra l'altro, votazioni in blocco sugli emendamenti. Con la decisione del Consiglio di Presidenza della Camera dei Deputati, durante la terza lettura della legge sul bilancio è stata consentita la votazione in blocchi degli emendamenti, comportando il rifiuto congiunto di tutti gli emendamenti proposti dallopposizione unitamente alle proposte della minoranza. Di conseguenza, con la seconda votazione sono stati adottati congiuntamente tutti gli emendamenti proposti dai deputati del partito di governo. Nella prassi parlamentare, era stato permesso nel passato di votare congiuntamente diversi emendamenti, solo se collegati da un elemento chiave comune. La maggior parte degli emendamenti proposti dallopposizione non aveva questo elemento chiave comune e talvolta si escludevano a vicenda. La controversia riguardava anche il fatto che ai parlamentari è stata negata la possibilità di presentare mozioni formali. Conformemente a quanto disposto dall'art. 184 del regolamento della Camera dei Deputati, ogni deputato ha il diritto di presentare una mozione formale e il presidente della Camera dei Deputati è tenuto a consentirgli di esercitare questo diritto.

Nel Resoconto integrale della 33a seduta della Camera dei Deputati della Repubblica di Polonia del 16 dicembre 2016 è stato riportato che due deputati del partito PO [Piattaforma Civica] hanno cercato di presentare mozioni formali in diverse occasioni, ma ciò non è stato loro concesso. Gravi dubbi di natura legale sono sorti anche in riferimento alla procedura di votazione. Nella Camera dei Deputati polacca tutte le votazioni sono palesi (art. 188, paragrafo 2, del regolamento della Camera dei Deputati). Poiché durante la votazione sulla legge sul bilancio non è stato possibile votare utilizzando il dispositivo per il conteggio delle preferenze, per soddisfare l'obbligo costituzionale e regolamentare circa la votazione palese, al resoconto integrale della seduta sarebbe stato necessario allegare un elenco di nomi con i risultati della votazione. Il 16 dicembre 2016, l'esito della votazione è stato determinato esclusivamente sulla base del risultato aggregato e non è stato allegato un elenco dei nomi dei votanti il che, pur consentito in determinate 
circostanze dal regolamento, non avrebbe dovuto avere luogo considerando l'importanza della legge sottoposta alla votazione che non consente il ricorso a questa scorciatoia procedurale.

Gravi riserve riguardo al corretto svolgimento del processo legislativo sono sorte anche in occasione degli ultimi lavori dedicati all'emendamento della legge che modifica il codice penale e alcune altre leggi. Nel capitolo 4 del regolamento della Camera dei Deputati è stata definita una procedura legislativa speciale, senza alcuna eccezione, per i progetti inerenti ai codici e alle modifiche agli stessi. Lart. 89 paragrafo 2 del regolamento della Camera dei Deputati stabilisce chiaramente che la prima lettura di un progetto di modifica a un codice può aver luogo non prima del quattordicesimo giorno successivo alla presentazione di detto progetto ai deputati. A sua volta, l'art. 95 paragrafo 1 del regolamento della Camera dei Deputati statuisce che la seconda lettura può avere luogo non prima del quattordicesimo giorno dalla data di presentazione ai deputati della relazione elaborata dalla Commissione Straordinaria. Considerando che tutte e tre le letture della legge analizzata si sono svolte in due giorni (15-16 maggio 2019), la legge è stata adottata in flagrante violazione del regolamento della Camera dei Deputati. Il disegno di legge del governo, presentato alla Camera dei Deputati (documento n. 3451) era volto a modificare il Codice penale, il Codice degli illeciti amministrativi, le disposizioni sull'implementazione del Codice penale, la Legge sull'applicazione delle sanzioni penali, il Codice di procedura penale (un progetto di 38 pagine complessivamente) e altre 5 leggi (un progetto di 2 pagine complessivamente, incluse due leggi volte a modificare il Codice penale). Sia il titolo del progetto di legge che i contenuti indicavano senza alcun dubbio il suo scopo: la modifica ai codici. Questo fatto non dipendeva dal parere del presidente della Camera dei Deputati, poiché la natura dell'atto giuridico, soggetto al dibattito, è determinata dai suoi contenuti e non dalla decisione autonoma dello Speaker?. Di conseguenza, la Camera dei Deputati era tenuta a procedere con i lavori sul disegno di legge proposto dal governo, volto a modificare il Codice penale e alcune altre leggi (documento n. 3451), rispettando sia le norme stabilite nel capitolo 4 del regolamento della Camera dei Deputati (art. 87 e seguenti) sia le scadenze ivi definite per le letture successive. La Camera dei Deputati, non osservando invece i tempi 
previsti per la prima e la seconda lettura (in entrambi i casi 14 giorni dalla data di presentazione del progetto ai deputati) e consentendo la mancata presentazione del progetto alla Commissione Straordinaria per l'istruttoria, ha visibilmente violato la procedura legislativa prevista dal regolamento della Camera dei Deputati per i progetti di modifica ai codici. A margine della presente analisi vale anche la pena sottolineare che secondo il parere del Difensore Civico e della Fondazione dell'Istituto di Diritto Penale di Cracovia (Fundacja Krakowskiego Instytutu Prawa Karnego), molte disposizioni della legge violano il principio costituzionale di proporzionalità, il principio uguaglianza davanti alla legge, il principio di separazione dei poteri, quello di equità nell'applicazione della legge, il principio di non discriminazione, l' obbligo di una legislazione adeguata e della definizione delle caratteristiche di un atto illecito (Z. Witkowski, M. Serowaniec, 2020, pp. 460-465).

Il ritmo dei lavori su molti progetti di legge svolti dalla Camera dei Deputati non solo non favorisce la qualità della legislazione, ma mina irrimediabilmente anche la dignità del parlamento. Lattività legislative è spesso condotta sotto la pressione dei tempi stretti, a tarda notte, senza possibilità di conoscere a fondo il progetto sia da parte dei parlamentari sia dei potenziali destinatari della nuova legge. Ad esempio, nel primo anno dellottava legislatura il processo legislativo nella Camera dei Deputati è durato in media 38 giorni, e nel primo trimestre del primo anno - solo 17 (IX Comunicato di Obywatelskie Forum Legislacji, 2016, p. 7). Nel secondo anno di legislatura, il periodo medio di lavori è durato 65 giorni e nel terzo anno 80 (XII Comunicato di Obywatelskie Forum Legislacji, 2018, p. 13). Attualmente, il procedimento di esame e di un'eventuale approvazione dei progetti di legge da parte del Senato dura in media 14 giorni. Nel primo anno della nona legislatura del Senato detto procedimento si concludeva in media in 11 giorni e nel primo trimestre del primo anno in soli tre giorni. Tuttavia, se si tiene conto del fatto che nel periodo analizzato ricadevano le vacanze parlamentari e che le date in cui si riunisce la Camera dei Deputati e il Senato di solito non coincidono, risulta che i senatori lavorano sulle leggi approvate dalla Camera dei Deputati per due, al massimo tre giorni. Ad esempio, è bastato solo un giorno affinché i senatori esprimessero il loro parere sulla Legge che modifica la legge sull'organizzazione dei tribunali ordinari e alcune 
altre leggi. La maggioranza del Senato, riunendosi di notte, ha respinto tutti gli emendamenti proposti dall'opposizione e ha approvato il progetto.

Considerando quanto sopra, destano giuste preoccupazioni i casi di un esame troppo rapido di progetti rilevanti, spesso complessi e che suscitano forti timori sociali. Negli ultimi quattro anni ciò è avvenuto, tra l'altro, nei casi di emendamenti alla legge sulla Corte suprema o delle proposte, da parte dei deputati, di emendamenti alle disposizioni che introducono la legge sull'organizzazione della Corte costituzionale e sulle modalità di procedimento dinanzi alla Corte costituzionale e la legge sullo status dei giudici della Corte costituzionale, nonché la legge sui tribunali ordinari. È significativo che queste importanti leggi costituzionali siano state modificate in base a progetti di legge presentati dai deputati o dal presidente della Repubblica, vale a dire senza consultazione, esame di diversi pareri e valutazione dell'impatto di un dato atto normativo. Spesso ci è voluto meno di un mese - dalla prima pubblicazione fino alla firma del Presidente - per promulgare una nuova legge.

\section{Nessun dibattito PARLAMENTARE SUI PROGETTI DI LEGGE}

Indubbiamente i tempi troppo brevi dei cambiamenti hanno un impatto negativo sulla certezza del diritto e limitano la possibilità di presentare riserve e obiezioni circa i progetti, nel caso o di leggere imperfezioni legislative o di questioni di importanza rilevante per lo Stato. Ciò riguarda particolarmente leggi molto ampie, complicate e volte a regolare una materia altamente complessa. Va sottolineato che la velocità accelerata dei lavori non dovrebbe servire a negare intenzionalmente, alla minoranza parlamentare, la possibilità di esprimere il parere e proporre eventuali soluzioni alternative (M. Zubik, 2018, p. 938-941). Non dovrebbe essere utilizzata neanche per impedire ad altre istituzioni statali e organizzazioni non governative di conoscere un dato progetto e sollevare obiezioni in caso di proposte difettose. La procedura di consultazione, pur ritenuta da qualsiasi maggioranza parlamentare onerosa o dispendiosa in termini di tempo, crea una sorta di meccanismo di allerta, consentendo di rilevare ed eliminare eventuali difetti di progetto nella fase appropriata del processo 
legislativo, quando tali difetti non sono ancora in grado di causare danni irreversibili (Z. Witkowski, M. Serowaniec, 2019, pp. 127-134).

Il corso dei lavori su numerosi progetti nell'attuale legislatura suggerisce purtroppo che l'impossibilità dell'opposizione di conoscere il contenuto del progetto e di presentare soluzioni alternative non sia un effetto collaterale, bensì una regola e uno scopo che orienta il procedimento adottato di formazione delle leggi. Questa tendenza, piuttosto pericolosa, include anche (in seguito alla modifica al regolamento della Camera dei Deputati in data 28 febbraio 2018) l'affidamento al Consiglio di Presidenza della Camera dei Deputati della competenza di determinare il limite delle domande da porre durante la prima lettura e discussione dei progetti di legge.

\section{Progetti governativi di Legge Vengono PRESENTATI COME PROPOSTE DEI DEPUTATI}

Il cambiamento importante riguarda anche il numero di leggi approvate in seguito alle proposte di legge presentate alla Camera dei Deputati dai membri della coalizione di governo. Nell'attuale legislatura è doppio rispetto alle legislature precedenti. L'aspetto ancora più rilevante è dato dal fatto che alcuni progetti proposti dai parlamentari si riferivano spesso ad aree finora regolate dal governo. In base alla proposta di legge d'iniziativa dei deputati sono state modificate ad esempio le regole di funzionamento della pubblica amministrazione e della Procura, della radio e della televisione, il Codice penale, il sistema della pubblica istruzione e persino l'ammontare del TFR nelle società statali. Nel corso del processo legislativo sono comparsi tuttavia molti indizi comprovanti la natura sostanzialmente governativa di quelle iniziative, proposte solo materialmente da parlamentari. Nel corso del procedimento legislativo i rappresentanti del Consiglio dei Ministri fungevano spesso da autori di un determinato progetto. Ad esempio, durante i lavori congiunti della Commissione parlamentare? per la pubblica istruzione, la scienza e la gioventù e della Commissione parlamentare? per le amministrazioni locali e le politiche regionali, dedicati al progetto di legge volto a modificare la legge sul sistema della pubblica istruzione e alcune altre leggi, è stata una rappresentante del Ministero della Pubblica Istruzione ad 
accettare o a respingere gli emendamenti presentati dai deputati. Inoltre, la ministra della Pubblica Istruzione, presente alla prima lettura nella Camera dei Deputati, ha ricordato: “...decine di conversazioni svoltesi nelle ultime settimane, durante gli incontri presso il Ministero della Pubblica Istruzione non solo con i sindacati, ma con tutte le organizzazioni che hanno ascoltato i nostri presupposti e discusso volentieri di queste soluzioni" (VI Comunicato di Obywatelskie Forum Legislacji, 2016, p. 3). Questa constatazione indica chiaramente che il progetto di legge è stato preparato dal governo, non da un gruppo di deputati. A confermare tale tesi sarebbe stata anche la mancata partecipazione, ai lavori su questi progetti, degli autori delle proposte o di altri parlamentari. Ad esempio, durante i lavori del Senato sulla proposta di legge d'iniziativa di un deputato, volta a modificare la legge sulla Corte costituzionale, alla riunione congiunta della Commissione Legislativa e della Commissione per i diritti dell'uomo, la legalità e le petizioni, il presidente della Commissione, vista la mancata presenza degli autori del progetto ha solamente constatato di aver mandato il relativo invito. In seguito alla proposta di un gruppo di senatori di non procedere in assenza di un rappresentante degli autori del progetto, il presidente ha indetto la votazione e con 7 voti a favore e 5 voti contrari è stato deciso che la riunione sarebbe continuata. In assenza dei proponenti, sono stati rappresentanti del Ministero della Giustizia a farne le veci. Inoltre, alcune delle proposte di legge presentate dai deputati erano precedentemente incluse nellelenco dei lavori legislativi e nella programmazione del Consiglio dei Ministri; tra di essi il progetto di legge volto a modificare la legge sulla Polizia e alcune altre leggi. Detto progetto, con la decisione del Consiglio dei Ministri del 5 gennaio 2016 è stato infine ritirato. La presentazione dei disegni di legge del governo come proposte dei deputati riduce i tempi dei lavori, il che è giustificato in situazioni di emergenza. Tuttavia, comporta una significativa limitazione del dibattito sulle soluzioni proposte e l'abbandono di consultazioni pubbliche. Le parti interessate, quali singoli cittadini o membri di organizzazioni civiche, non possono in pratica esprimere alcun parere o sollevare riserve circa le modifiche proposte (Z. Witkowski, M. Serowaniec, 2020, pp. 460-465).

$\mathrm{Va}$ anche notato che numerosi disegni di legge partiti dal governo sono stati 'svelati' solamente all'inizio di lavori della Camera dei Deputati. 
In pratica, visto il numero rilevante di progetti soggetti a esame, si fa fatica ad accedere a informazioni dettagliate sui tempi o sulla natura dei lavori svolti all'interno del governo. In alcuni casi i cittadini scoprivano l'esistenza di un dato progetto governativo solo quando veniva sottoposto all'esame del parlamento. È difficile da stabilire il tempo esatto dedicato dal governo al progetto di legge volto a modificare la legge sull'Istituto Polacco per la Memoria Nazionale - Commissione per la lotta ai reati commessi contro la nazione polacca e la legge sulla responsabilità dei soggetti collettivi di fronte ad atti illeciti. I primi documenti legati a detto progetto sono stati messi a diposizione sulla piattaforma del Procedimento Legislativo del Governo in data di presentazione dello stesso alla Camera dei Deputati, ossia il 27 giugno. Il progetto di modifica e l'analisi d'impatto della regolazione sono datati il 26 giugno, ma il Consiglio dei Ministri ha deciso solo l'11 luglio, vale a dire due settimane dopo l'approvazione della legge, di mandarlo alla Camera dei Deputati! Mettendo insieme i progetti di legge 'nascosti' in questo modo, le leggi formate con il procedimento abbreviato e i disegni di legge d'iniziativa dei deputati, risulterebbe che circa il 30\% delle leggi è entrata in vigore senza alcuna discussione pubblica. Le riunioni delle commissioni parlamentari difficilmente fungono da luogo di dibattito pubblico e aperto, dove ogni parte interessata possa presentare il proprio parere sui progetti in esame: i cittadini hanno un accesso limitato alle sedute delle commissioni della Camera dei Deputati e a ciò si aggiunga l'abbandono delle audizioni pubbliche e la limitazione del tempo di intervento durante le sedute della Camera dei Deputati.

\section{Consultazioni PuBbliche E ALTRE ATtività DI CONSULTAZIONE}

Esistono almeno due motivi per i quali le autorità dovrebbero provvedere a una consultazione pubblica appropriata, aperta e disponibile a tutti i soggetti interessati sui progetti di atti giuridici. Una consultazione ben condotta potrebbe aiutare gli organi dell'amministrazione pubblica a prendere decisioni giuste, rappresentando una fonte di informazioni tecniche aggiuntive nonché uno strumento di verifica approfondita e oggettiva della qualità del progetto 
di legge e dell'analisi sull'impatto della regolazione, elaborata dall'autore di un dato progetto. D’altro canto, la consultazione potrebbe offrire ai soggetti e alle istituzioni coinvolti un valore aggiunto nel processo decisionale, facilitando in futuro l'implementazione e il rispetto della legge in oggetto. Lanalisi della prassi legislativa del governo ci fornisce invece numerosi esempi di limitazione intenzionata del ruolo delle consultazioni. I tempi medi dedicati alla consultazione pubblica oscillano attorno a soli 13 giorni. In un lasso di tempo così breve la maggior parte dei soggetti interessati, che non rappresentino istituzioni importanti, non ha mezzi necessari per conoscere in dettaglio i progetti di legge, consultarli con propri esperti e preparare commenti e pareri tecnici adeguatamente formulati.

Molto raramente si ricorre a una pre-consultazione delle parti interessate? che consentirebbe di conoscere il parere degli esperti in un'iniziale fase concettuale del lavoro legislativo. Nel caso di proposte parlamentari che non sono sottoposte a procedure di consultazione pubblica o di progetti del governo, controversi o oggetto di consultazioni insufficienti, le parti interessate cercano di presentare le loro argomentazioni durante le riunioni delle commissioni parlamentari. Purtroppo, molto spesso le loro voci vengono ignorate, specialmente se si tratta di unopinione dissenziente rispetto alla posizione del governo. Va anche notato che l'opinione dei legislatori è accettata quando riguarda questioni strettamente tecniche. Altri commenti sostanziali sono di solito trascurati. Una delle conseguenze di tale prassi è la promulgazione di atti giuridici difettosi, che spesso, e rapidamente, devono essere rettificati. In questo contesto, vale la pena di ricordare che la legge sulla Corte Suprema dell'8 dicembre 2017, preparata dal Presidente, è stata modificata nel 2018 ben sette volte dopo essere entrata in vigore nel suo testo originario. I lavori della Commissione dedicati al progetto di legge del presidente si sono svolti in unatmosfera molto nervosa. Alle riunioni della Commissione per la Giustizia e i Diritti dell'uomo avvenute nei giorni 28-30 novembre e il 6 dicembre 2017, hanno partecipato i rappresentanti del Consiglio Nazionale della Magistratura, del Difensore Civico, del Comitato Helsinki per i Diritti Umani, dell'Associazione Iustitia e della Protezione Civile e Sociale "Krajowy Sztab Ratownictwa Społecznej Sieci Ratunkowej”. Per la maggior parte, hanno formulato opinioni critiche sul progetto, accusandolo di una 
palese contraddizione con i principi fondamentali dell'ordinamento politico della Repubblica di Polonia. Tuttavia, ciò non ha avuto alcun impatto né sui contenuti del progetto né sullo svolgimento dei lavori della Commissione. Le loro opinioni sono state ignorate, diventando persino oggetto di attacchi da parte della maggioranza parlamentare. Durante la quarta riunione (6 dicembre 2017), a un rappresentante del Consiglio Nazionale della Magistratura è stato negato più volte il diritto di voto. In queste circostanze gli esperti della Camera dei Deputati non hanno avuto l'opportunità di partecipare in modo concreto alla controversia legale. La loro partecipazione è stata marginale e ridotta all'introduzione di modifiche strettamente tecniche. Nel gennaio 2018, la Corte dei Conti ha pubblicato una relazione sulla valutazione dell'impatto dei processi legislativi del governo. Analizzando il lavoro di quattro ministeri, negli anni 2015-2016, la Corte dei Conti ha constatato che: "I vantaggi offerti dalle consultazioni pubbliche sono stati sfruttati in misura limitata. Sebbene i requisiti formali riguardanti le modalità di svolgimento del processo di consultazione pubblica, specificati nel regolamento del Consiglio dei Ministri, siano stati per lo più soddisfatti, molte raccomandazioni in merito, indicate nei documenti non soggetti allobbligo di consultazione, non sono state adottate. La pratica di abbreviare le scadenze per la presentazione dei pareri, di limitare l'ambito di consultazione solo alla fase di esame di un progetto pronto, l'uso di un ventaglio ristretto di forme di consultazione, la mancata risposta o una risposta non immediata alle riserve sollevate, possono indicare che le consultazioni sono trattate come un dovere che rallenti il procedimento di formazione delle leggi e che non rappresenti una fonte di informazioni rilevanti" (Supreme Audit Office, 2018, p. 3-5). Nella presente legislatura del il mancato rispetto delle norme sulla consultazione sui progetti di legge con le parti interessate è diventato purtroppo una prassi.

\section{Conclusioni}

Attualmente, le 'leggi' in Polonia vengono spesso approvate furtivamente, in un lasso di tempo inaccettabile, senza un dibattito pubblico oggettivo e concreto. Tutti gli sforzi di sottoporre l'azione legislativa a una riflessione seria risultano infruttuosi sebbene le autorità possano attingere al un ricco 
bagaglio e patrimonio scientifico nel campo del diritto costituzionale e di altre discipline del diritto polacco, sviluppatosi negli ultimi venticinque anni. Le opinioni dei dipartimenti legali della Corte suprema, della Camera dei Deputati e del Senato, dei soggetti sociali come la Fondazione Batory o il Comitato Helsinki per i Diritti Umani, se negative o contrarie all'adozione di determinate leggi, non vengono intenzionalmente prese in considerazione.

Tale chiusura del governo in carica e della sua maggioranza verso qualsiasi forma di dibattito e di confronto impedisce anche il raggiungimento di un compromesso politico con lopposizione. Un esempio eclatante del fenomeno qui analizzato è rappresentato dall'infelice idea del presidente della Repubblica di un referendum costituzionale consultivo, non previsto dalla Costituzione polacca; la proposta in oggetto è stata chiaramente in contrasto all'assiologia e alla Costituzione della Repubblica di Polonia in vigore dal 1997 (Z. Witkowski, A. Bień-Kacała, 2012, p. 536).

La macchina legislativa parlamentare 'rigurgita' leggi coniate sulla base di un chiaro input governativo, e successivamente la macchina esecutiva implementa tali leggi, senza tenere conto dei principi e degli standard legislativi adottati nel mondo democratico (Z. Witkowski, 2019, p. 137-138). Nessun soggetto, dellattuale partito al potere, prende in considerazione il fatto che la legge così formata e implementata diventa una farsa legale, in quanto - come dicevano già gli antichi Romani - da un atto illecito non può nascere un diritto (Ex iniuria ius non oritur). In questo modo si sta addirittura creando un modello di 'legislazione indecente' (M. Wyrzykowski, 2017, p. 9).

\section{Bibliografia}

Antoszewski, A. (2018). Demokracja nieliberalna jako projekt polityczny, Przegląd Europejski 2, p. 14. DOI: 10.5604/01.3001.0013.0788.

Fundacja im. Stefana Batorego. IX Comunicato di Obywatelskie Forum Legislacji sulla qualità del processo legislativo in base allanalisi svolta nei giorni 11 settembre - 10 dicembre 2016. Disponibile online (13.12.2020): http://www.batory. org.pl/upload/files/Programy\%20operacyjne/Odpowiedzialne\%20Panstwo/ Komunikat\%20z\%20IX\%20obserwacji.pdf. 
Fundacja im. Stefana Batorego. VI Comunicato di Obywatelskie Forum Legislacji. Analisi del procedimento di formazione delle leggi nei giorni: 16 novembre 2015-15 febbraio 2016. Disponibile on-line (17.12.2020) http://www.batory.org.pl/upload/ files/Programy\%20operacyjne/Odpowiedzialne\%20Panstwo/Komunikat\%20 VI\%20obserwacja.pdf.

Fundacja im. Stefana Batorego. XII Comunicato di Obywatelskie Forum Legislacji sulla qualità del processo legislativo in base allanalisi svolta nei giorni 16 maggio - 15 novembre 2018. Disponibile on-line (13.12.2020) http://www.batory.org.pl/ upload/files/Programy\%20operacyjne/Forum\%20Idei/XII_Komunikat_OFL.pdf.

Makowski, G. Nowelizacje ustawy o Instytucie Pamięci Narodowej i siedem grzechów głównych państwa PiS. Disponibile on-line (12.12.2020) http://www.batory.org.pl/ upload/files/Programy\%20operacyjne/Forum\%20Idei/Grzechy_PiS_nowelizacje_ IPN.pdf.

Supreme Audit Office. Informacja o wynikach kontroli, Dokonywanie oceny wpływu $w$ ramach rzadowego procesu legislacyjnego. Disponibile on-line (13.12.2020) https://www.nik.gov.pl/plik/id,16190,vp,18712.pdf.

Witkowski, Z. i Serowaniec, M. (2019). Constitutional conditions of the legislative process in Poland - theory vs practice, Kobe University Law Review 52, p. 127-134. DOI:10.30970/jcl.4.2019.2.

Witkowski, Z. (2015). Siedem grzechów głównych polskiej klasy politycznej wobec wyborców, wyborów i prawa wyborczego, Toruń: Wydawnictwo TNOiK Dom Organizatora. ISBN 9788372857743.

Witkowski, Z. (2019). Kilka uwag w kwestii kultury prawnej i politycznej współczesnych elit rzadzacych $w$ Polsce ( $w$ kontekście zmian ustroju władzy sądowniczej), w: [in:] Alena Krunková (ed.), Organizácia súdnej moci v Pol’skej republike, Českej republike a Slovenskej republike (p. 137-138), Koszyce: Vydavatel'stvo ŠafárikPress UPJŠ v Košiciach. ISBN 9788081527401.

Witkowski, Z. i Bień-Kacała, A. (2012). „Mania ustawodawcza”-przewlekła choroba polskiego parlamentu $i$ metody jej leczenia, w: Nauki penalne wobec szybkich przemian socjokulturowych. Księga jubileuszowa Profesora Mariana Filara (p. 536), Toruń: Wydawnictwo Adam Marszałek. ISBN 9788377804650.

Witkowski, Z. i Serowaniec, M. (2020). Standards of "indecent legislation" in Poland, w: R. Arnold, I. Daneliene (eds.), The concept of democracy as developed by constitutional justice (p. 460-465), Vilnius: The Constitutional Court of the Republic of Lithuania. ISBN 9789955688471.

Wyrzykowski, M. (2017). Obrona Konstytucji RP. Kwartalnik o prawach człowieka 3-4, p. 9. ISSN 2300-2891. 
Wyrzykowski, M. (2020). Antyzasada nieprzywoitej legislacji (casus nowelizacji ustawy o Instytucie Pamięci Narodowej), w: A. Gajda, K. Grajewski, A. RytelWarzocha, P. Uziębło, M.M. Wiszowaty (eds.), Konstytucjonalizm polski. Refleksje z okazji jubileuszu 70-lecia urodzin i 45-lecia pracy naukowej Profesora Andrzeja Szmyta (p. 721-722), Gdańsk: Wydawnictwo Uniwersytetu Gdańskiego. ISBN 9788378659693.

Zubik, M. (2018). Parlament przed sądem praw człowieka, w: M. Serowaniec, A. Bień-Kacała, A. Kustra-Rogatka (eds.), Potentia non est nisi da bonum: edizione giubileo dedicata al prof. Zbigniew Witkowski (p. 938-941), Toruń: Wydawnictwo TNOiK Dom Organizatora. ISBN 9788372858511.

Zubik, M. (2020). Sejm VIII kadencji. Ostatnia kadencja Rzeczypospolitej czy pierwsza „dobrej zmiany”?, w: A. Gajda, K. Grajewski, A. Rytel-Warzocha, P. Uziębło, M.M. Wiszowaty (eds.), Konstytucjonalizm polski. Refleksje z okazji jubileuszu 70-lecia urodzin i 45-lecia pracy naukowej Profesora Andrzeja Szmyta (p. 731-732), Gdańsk: Wydawnictwo Uniwersytetu Gdańskiego. ISBN 9788378659693. 
